\title{
VINCENT GÉLINAS-LEMAIRE, Le récit architecte. Cinq aspects de l'espace
}

\section{Luana Doni}

\section{(2) OpenEdition}

\section{Journals}

\section{Edizione digitale}

URL: https://journals.openedition.org/studifrancesi/32486

DOI: 10.4000/studifrancesi.32486

ISSN: 2421-5856

\section{Editore}

Rosenberg \& Sellier

\section{Edizione cartacea}

Data di pubblicazione: 1 août 2020

Paginazione: 461-462

ISSN: 0039-2944

\section{Notizia bibliografica digitale}

Luana Doni, «VINCENT GéLINAS-LEMAIRE, Le récit architecte. Cinq aspects de l'espace», Studi Francesi

[Online], 191 (LXIV | II) | 2020, online dal 01 septembre 2020, consultato il 18 septembre 2021. URL:

http://journals.openedition.org/studifrancesi/32486 ; DOI: https://doi.org/10.4000/studifrancesi. 32486

Questo documento è stato generato automaticamente il 18 septembre 2021.

\section{(c) (i) (9)}

Studi Francesi è distribuita con Licenza Creative Commons Attribuzione - Non commerciale - Non opere derivate 4.0 Internazionale. 


\title{
VINCENT GÉLINAS-LEMAIRE, Le récit architecte. Cinq aspects de l'espace
}

\author{
Luana Doni
}

\section{NOTIZIA}

VINCENT GÉLINAS-LEMAIRE, Le récit architecte. Cinq aspects de l'espace, Paris, Classiques Garnier, 2019, 243 pp.

1 Nel racconto, l'importanza dello spazio è fondamentale; esso non ne costituisce soltanto lo sfondo, ma anche la forma, la direzione e, talvolta, il senso stesso. Lo studio proposto da Vincent GÉLINAS-LEMAIRE consiste nell'identificare i meccanismi fondamentali nella rappresentazione dello spazio nella letteratura, seguendo le orme di quanto delineato da Gérard Genette nel suo Discours du récit.

2 L'analisi viene suddivisa dall'autore in cinque capitoli riguardanti le varie prospettive adottate in materia di spazio e di racconto.

3 L'aspetto geometrico dello spazio («Aspect géométrique de l'espace», pp. 17-47) costituisce il punto di partenza dell'indagine, poiché risulta particolarmente adatto a cogliere le varie tipologie spaziali e narrative. Il geometrico, come sostiene MerleauPonty, è uno dei modi possibili di apprendimento del mondo, uno strumento polivalente che si integra alla voce narrativa. L'aspetto geometrico è ciò che delimita lo spazio e che lo intreccia alla materialità fisica del personaggio. Il romanzo La Jalousie di Alain Robbe-Grillet viene utilizzato come esempio, in quanto tutti i dettagli riguardanti la vita sociale del protagonista sono racchiusi in un sistema cifrato di forme geometriche atto a dimostrare la prova oggettiva del tradimento della moglie.

Quello che viene definito l'«Aspect localisé de l'espace» (pp. 49-90) coincide con la rintracciabilità del luogo data la sua appartenenza a un'area geografica limitata o alla presenza di caratteristiche specifiche. Lo studio della poetica narrativa permette di concepire il luogo come movimento, come trasformazione da uno spazio informe, a uno denso di significato e riconoscibile. Dalla Venezia di Thomas Mann alla Londra di 
Dickens, passando per la Verona di Shakespeare, si scopre che l'architettura determinante di alcuni luoghi simbolo è essenzialmente quella che riguarda la loro resa letteraria. Il potere evocativo di alcuni toponimi, come quelli sopracitati, restituisce allo spazio un contenuto aprioristico che ne trascende quello a posteriori.

5 L'aspetto allegorico dello spazio («Aspect allégorique de l'espace», pp. 100-144), al quale è consacrato il terzo capitolo, è definibile attraverso il confronto parallelo con i personaggi del racconto, con una qualità morale, con un oggetto, astratto o tangibile che sia. L'allegoria è qui concepita come una relazione apparente tra due termini, relazione a volte ambigua, mobile e fuorviante. Entrando nello specifico del testo, l'autore propone tre esempi diversi, accomunati dalla presenza dello spazio come quadro di una relazione allegorica. Il primo esempio, quello definito di allegoria implicita, è rappresentato dal Roman de la Rose e dalle vicende del suo protagonista che, avvicinandosi alle mura che circondano un frutteto, ne scopre la lussuriosa natura popolata da nobili figure. Se i personaggi del poema sono inseriti in quanto simboli dell'amor cortese, il frutteto e le sue mura offrono un'interpretazione più ambigua: il muro è sì lo schermo sul quale si proiettano le allegorie rappresentanti qualità intangibili, ma esso è anche un perimetro geometrico, un ostacolo per lo sguardo e i movimenti del personaggio. Il secondo esempio, quello di allegoria esplicita, fa riferimento a Le Livre de la Cité des Dames di Christine de Pizan, nel quale la poetica spaziale è interamente votata alla contestazione di un argomento, quello che incoraggia gli uomini a essere oltraggiosi nei confronti delle donne. La città è dunque costruita sull'evocazione delle qualità femminili, e la stessa cosa accade alla presenza dei toponimi; la Cité, infatti, è situata nel regno di Féminie e sul Champ des Lettres. L'ultimo esempio, quello relativo allo spazio come soggetto allegorico, è tratto da Le Rivage des Syrtes di Julien Gracq, paradigma della gestione di un racconto da parte dei suoi spazi. Il protagonista, Aldo, è infatti schiavo di pulsioni geografiche che lo attirano e lo trascinano verso la catastrofe.

6 Il capitolo riguardante l'aspetto dinamico dello spazio («Aspect dynamique de l'espace», pp. 145-185) fa riferimento a tutti quei casi in cui lo spazio entra in relazione diretta con il personaggio e al ruolo che quest'ultimo gioca nella rappresentazione dell'ambiente che lo circonda. Per il geografo Yi-Fu Tuan, il corpo è un oggetto fisico direttamente proporzionale al suo ambiente ed è strumento di conoscenza del mondo, nonché unica prospettiva sul mondo. Le interazioni tra soggetto e mondo, nella loro varietà e importanza, sono affrontate nel capitolo attraverso il dispositivo teatrale, in grado di fornire un aiuto alla descrizione delle strategie poetiche che costruiscono lo spazio attraverso la mediazione dell'individuo.

7 L'ultima riflessione del volume è dedicata all'aspetto tecnico dello spazio («Aspect technique de l'espace», pp. 187-225), ossia la realtà sensibile dello spazio, la volontà di concepire l'arte e la scienza come intrinsecamente spaziali. Convocando a titolo di esempio due classici della letteratura come Bouvard et Pécuchet e Robinson Crusoe, l'autore solleva la questione del divario tra verosimiglianza e attendibilità scientifica. 